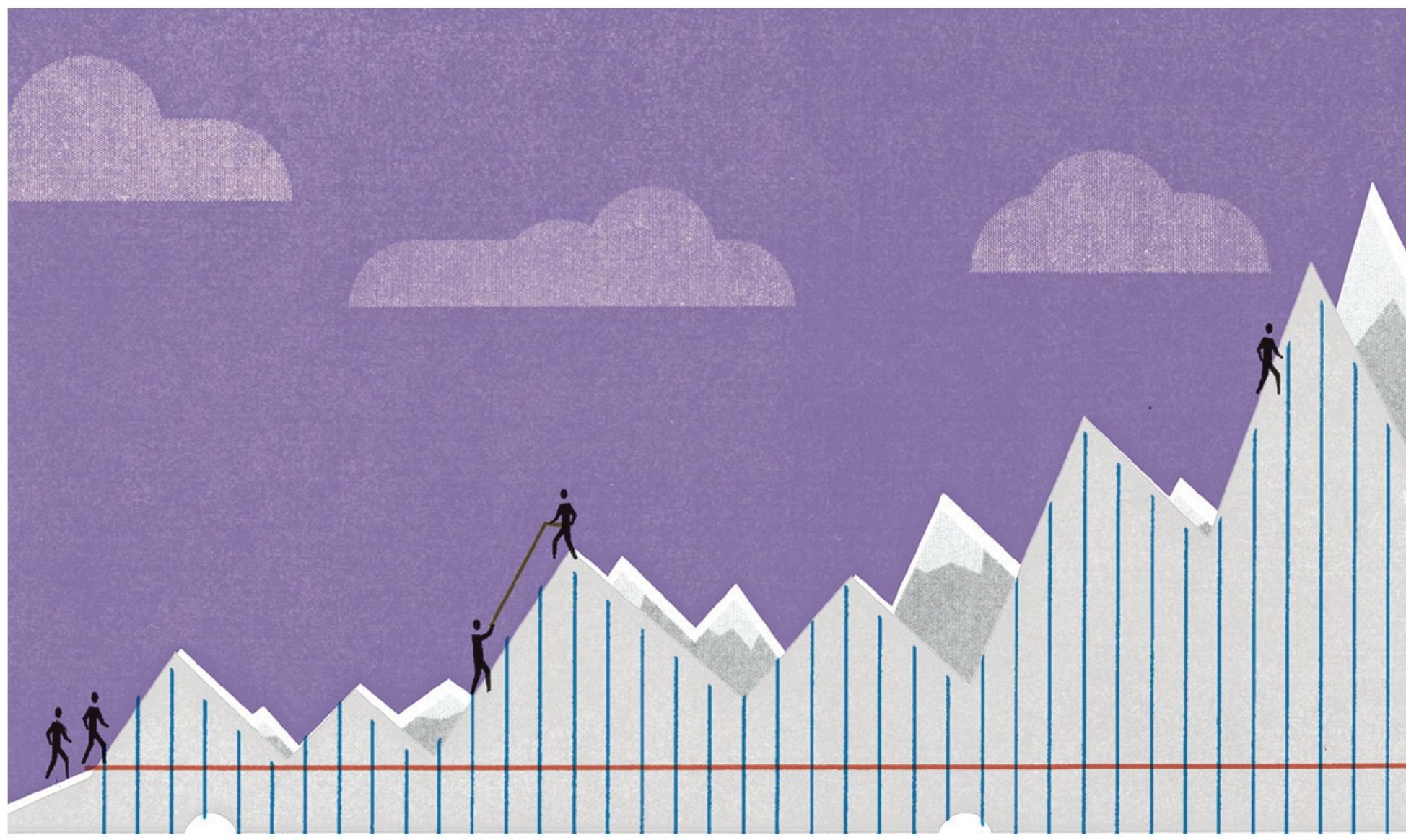

\title{
Honour the helpful
}

\section{Alexander Oettl presents evidence that scientists who share advice and expertise enhance their colleagues' productivity.}

$\mathrm{W}$ hat kind of scientist does a department want to hire? The investigator who churns out high-impact papers and travels around the world giving seminars? Or someone with an average publication record who is always discussing other people's work with them, attending seminars and providing feedback on colleagues' papers?

Most departments would probably hire the first one and not think twice. I believe the choice is not so straightforward. Traditionally, science has been an individual pursuit, in which people were valued for their personal output and achievements. Discovery increasingly relies on teamwork and yet scientists are still judged only by what they themselves accomplish.

Throughout my career I've had excellent colleagues. Even if they weren't participating directly in a project, they provided crucial advice that shaped an experiment, gave thoughtful criticisms of manuscripts and shared data and expertise with anyone who asked. Such people are often thanked in the acknowledgments of papers, not listed as co-authors. But this quality - I call it 'helpfulness' - isn't measured in job applications nor is it considered by hiring committees.

It should be. By reviewing the acknowledgements in immunology papers since 1950, I have found that when principal investigators (PIs) who were frequently thanked by others died unexpectedly, the quality of the papers of their collaborators dropped. In contrast, the quality of co-authors' work changed little after the passing away of PIs who were not particularly helpful to others.

To me, this hints that we need to reevaluate what makes some scientist 'stars', to make room for those who may have fewer individual achievements but are a major source of support and feedback for the people around them. Especially nowadays, when it can seem harder than ever to spare time to help someone else, such collegiality should be encouraged. It benefits the entire scientific enterprise.

\section{WITH THANKS}

I examined the acknowledgements from the Journal of Immunology - the society journal of the American Association of Immunologists (impact factor 5.788) - from 1950 to 2007 (more than 50,000 papers), using software to note who was thanked, and for what (A. Oettl Manage. Sci. 58, 1122-1140; 2012). To find investigators who probably died while still actively working and publishing - and thus can act as a quasi-natural experiment - I extracted obituaries from more than 400,000 immunology articles from Web of Science and the American Association of Immunologists newsletter. I then looked for people who died within 50 years after publishing their first paper, and checked that they and their co-authors were actively publishing up to the PI's death. 


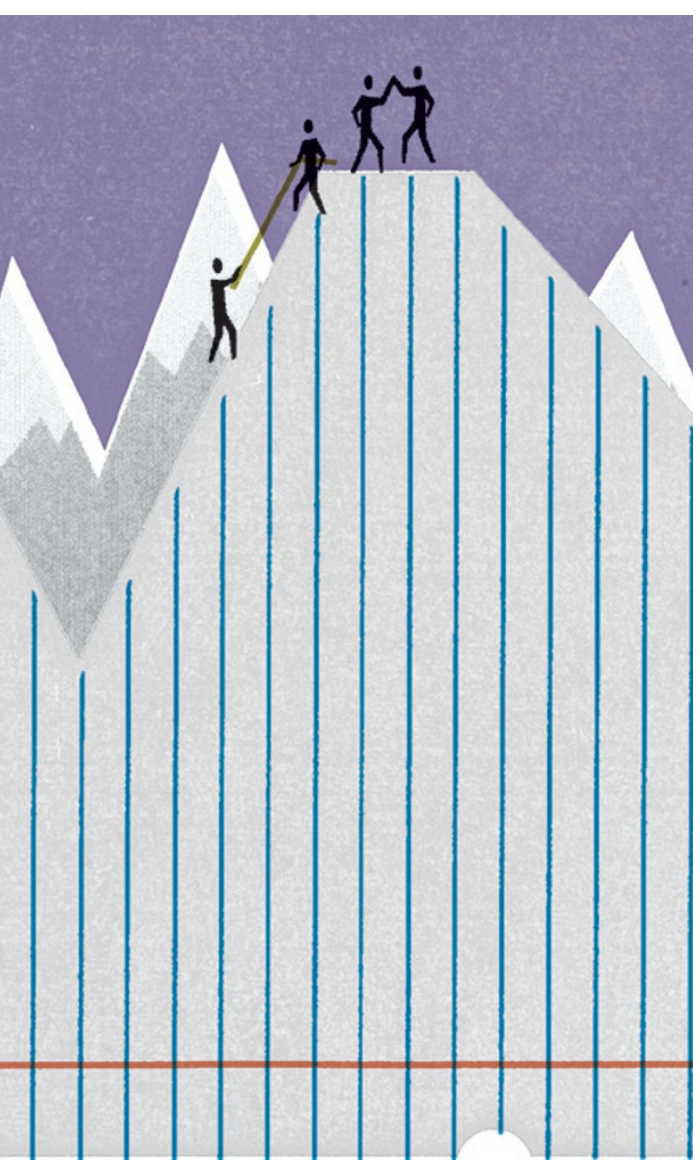

I found 149 PIs (either first or last authors) who had died in mid-career. Of these, 63 were in the top $20 \%$ of people thanked in all acknowledgements for the year in at least one year of their publishing career. I 을 call this group 'helpful'. Thirty-five of the PIs in this group were also highly productive, defined as being in the top $5 \%$ for the number of annual citations and high-impactfactor immunology publications. I similarly divided the less helpful PIs into productive and less productive categories.

I found that co-authors of helpful PIs experienced a similar decrease in the number of papers published after the death of those PIs as did the co-authors of less helpful and less productive PIs (see 'When help fades'). But co-authors of the helpful PIs experienced a distinct decline in the quality of their publications compared with the less helpful groups - as judged on the impact factors of the journals in which they published. Co-authors of the helpful and most productive PIs experienced a $22 \%$ decline $(P<0.01)$, whereas those of the helpful but less productive PIs saw a $20 \%$ decline. In some cases, the dip lasted for more than five years.

Co-authors of highly productive and helpful PIs received $21 \%$ fewer citations, by a 2010 cut-off, for work published after the PI's death. Citations dropped by $28 \%$ on average over the same time period for co-authors of helpful but not particularly productive PIs.

In contrast, co-authors of the 17 PIs who were not particularly helpful but had published many papers in high-impact journals, did not experience any statistically significant decline in the quality or number of their publications or in the number of citations received after their colleague's death. This may seem as if working with unhelpful colleagues means that a scientist's subsequent work is saved from being affected. But these results also suggest that had these co-authors worked with a helpful PI over the same period, their productivity might have been even greater.

The impact of a death was particularly profound on co-authors of PIs who were helpful with conceptual feedback, such as advice and criticism. Perhaps this is because such services are harder to replace than technical expertise, reagents and other study materials.

A good question is: if those helpful PIs with average publication records have such a positive impact on their colleagues' experiments, why aren't they more successful themselves? But there are many instances in science where these qualities don't go hand in hand - for example, many referees can

\section{WHEN HELP FADES}

After 'helpful' PIs die in mid-career, their co-authors experience a dramatic decline in high-impact publications and citations. In contrast, there is no significant change in these for co-authors of 'unhelpful' Pls after their death (error bars indicate 95\% confidence intervals).

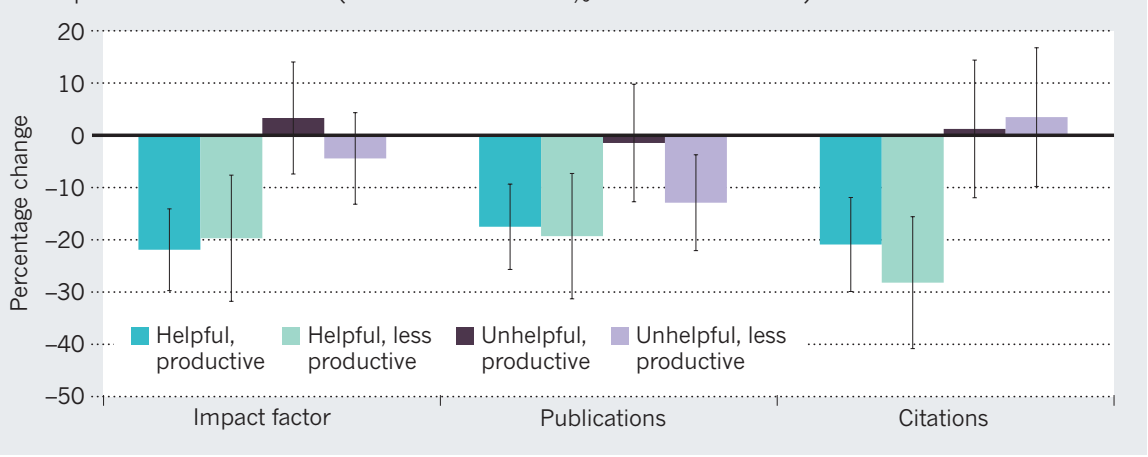

have a substantive, positive effect on the quality of a manuscript even though they may not have been able to produce a work of similar quality themselves.

Of course this study has limitations. It examines acknowledgements in just one journal within a single biomedical field. In addition, the deaths

"Collegiality should be encouraged. It benefits the entire scientific enterprise." I record are by no means exhaustive, as I must rely on secondary data sources. Ideally, I would examine whether there is an increase in work quality after the formation of a relationship with a helpful PI, rather than the effects of the loss of that benefit. But this would require an elaborate randomized field experiment involving forced co-authorship - hardly a realistic possibility.

\section{TEAM SPIRIT}

My results suggest that scientists who are helpful have a major impact on their colleagues' careers - and have been undervalued by a scientific enterprise that rewards individual achievement above all else.

It is time to look more closely at what qualities we value most in scientists. Researchers who generate numerous high-impact papers may have little time to discuss problems, criticize manuscripts or mentor students. Those who produce a stream of average papers may have a much more positive impact on the careers of the people around them. Researchers looking for collaborators may sometimes opt for a helpful colleague who is not a major force in their field over a rock-star scientist who rarely replies to e-mails.

So hiring committees should look beyond an applicant's publication record. They should read the recommendations of peers and look for signs that the individual might influence departmental dynamics in a positive way.

Perhaps we should even develop a metric that measures helpfulness, such as average acknowledgments per year? And funding agencies could give 'helpfulness' awards for generosity that has benefited the field at large, to encourage scientists to help others.

Of course, not every scientist is able to be more helpful. For those who have trouble interacting with others, say, trying to change their natural inclinations and personality will be a losing battle. But science does need to change its reward structure so that researchers who do a lot for others are not penalized.

Alexander Oettl is an assistant professor at the Georgia Institute of Technology Scheller College of Business, Atlanta, Georgia 30308, USA.

e-mail:alex.oettl@scheller.gatech.edu 